\title{
Efficacy and safety of sorafenib versus apatinib in the treatment of intermediate and advanced hepatocellular carcinoma: a comparative retrospective study
}

This article was published in the following Dove Press journal: OncoTargets and Therapy

\author{
Yizhuo Wang ${ }^{1,2}$ \\ Qing Gou' \\ Rongde $\mathrm{Xu}{ }^{\prime}$ \\ Xiaoming Chen' \\ Zejian Zhou'
}

'Department of Interventional Oncology, Guangdong Academy of Medical Sciences, Guangdong General Hospital, Guangzhou, Guangdong, People's Republic of China; ${ }^{2}$ Medical College Shantou University, Shantou, Guangdong, People's Republic of China
Correspondence: Zejian Zhou Guangdong Academy of Medical Sciences, Guangdong General Hospital, 106

Zhongshan Road, Guangzhou, Guangdong 5 I 0080, People's Republic of China

Tel +86 I392608 9188

Email zhzejian@I26.com
Objective: To compare the efficacy and safety profiles of sorafenib and apatinib in patients with intermediate- and advanced-stage hepatocellular carcinoma (HCC).

Methods: This was a single-center, retrospective study where we collected the clinical data of 72 patients, diagnosed with intermediate or advanced HCC from January 2014 to December 2016. Depending on the treatment received, 38 patients were categorized into group S (sorafenib group) and 34 into group A (apatinib group). The patients in group A received the initial recommended dose of $750 \mathrm{mg}$ once daily (QD), which was reduced to $250 \mathrm{mg}$ QD in the case of any class 3 or 4 adverse event (AE). Sorafenib was administered orally $400 \mathrm{mg}$ twice daily (BID), and dose was modified to $400 \mathrm{mg}$ or $200 \mathrm{mg}$ QD in the case of grade 3 or 4 AEs. The median overall survival (OS), progression-free survival (PFS), and AEs reported in the two groups were analyzed and compared.

Results: Among the 38 patients treated with sorafenib, one patient had complete response (CR), 5 patients had partial response (PR), and 10 patients had stable disease (SD), and among the 34 patients treated with apatinib, 6 patients had PR and 7 patients had SD with no cases of CR. PFS in group $\mathrm{S}$ was significantly longer compared with that in group A (7.39 vs 4.79 months, respectively, $P=0.031)$. Similar observations were made for median OS (10.4 months in group $\mathrm{S}$ vs 7.18 months in group $\mathrm{A}, P=0.011)$. However, there was no significant difference in the objective response rates (ORRs) among the study population ( 15.7 vs $17.6 \%, P=0.829$ ). Common AEs in group $\mathrm{S}$ included hand and foot syndrome (HFS) and diarrhea, whereas common AEs in group A included hypertension, proteinuria, and increased transaminase.

Conclusion: Our study showed promising clinical outcome with apatinib, but the sorafenib group exhibited better clinical efficacy with no significant difference in safety profile.

Keywords: observational, HCC, sorafenib, apatinib, overall survival, progression-free survival

\section{Background}

Hepatocellular carcinoma (HCC) ranks as the fifth most common solid tumor and among top 3 causes of global cancers' related mortality. ${ }^{1,2}$ Of all the global cases of $\mathrm{HCC},>75 \%$ occur in the Asia-Pacific region ${ }^{3,4}$ with China leading with $>50 \%$ of the reported cases. ${ }^{3}$ Due to high incidence of HCC in China ( $>30$ cases/100,000 population per year), ${ }^{1}$ it is very important to assess the efficacy and safety of newer therapeutic agents for HCC in the high-risk population. As HCC is a highly vascularized tumor characterized by the overexpression of vascular endothelial growth factor (VEGF) and 
platelet-derived growth factor (PDGF) leading to increased metastatic potential, ${ }^{4}$ inhibitors of VEGF and PDGF signaling pathways are the frequently applied agents in HCC.

The treatment for HCC includes surgical and nonsurgical methods. Currently, multidisciplinary treatment strategies are followed for HCC in China that include radical resection, liver transplantation, palliative surgery, transcatheter arterial chemoembolization (TACE), radiotherapy, immunotherapy, chemotherapy, molecularly targeted therapy, and traditional Chinese herbal medicine. ${ }^{5}$

Sorafenib, a multikinase inhibitor that inhibits tumor angiogenesis and cell proliferation, ${ }^{6}$ has been recommended as the gold standard treatment for intermediate and advanced $\mathrm{HCC}$ by at least 8 major guidelines for HCC including Barcelona-Clinic Liver Cancer (BCLC) and National Comprehensive Cancer Network (NCCN) guidelines. ${ }^{7}$ Evidence of sorafenib showing significantly greater survival compared with placebo is reported in multiple studies. ${ }^{3,8}$ The SHARP study, sorafenib reported an increase in the median survival and the time to radiologic progression by 3 months in US and European patients treated with sorafenib in comparison to placebo. $^{8}$ Similarly, the ORIENTAL study conducted in the Asia-Pacific reported a significantly longer overall survival (OS) in sorafenib-treated patients. ${ }^{3}$ Despite sorafenib been approved for advanced HCC, it is not recommended routinely in clinical practice owing to its modest survival benefits and high treatment cost. ${ }^{9}$ Several markers such as VEGFR and c-Kit, etc have been shown to predict OS but have not reported the response to sorafenib in advanced HCC. ${ }^{10}$ Hence, patients progressing after sorafenib treatment or those not able to tolerate sorafenib have limited treatment options and a large unmet need still exists.

Apatinib, a selective inhibitor of VEGFR-2 and mild c-Kit and c-Src tyrosine kinase inhibitor, has shown encouraging results in the treatment of a variety of solid tumors. ${ }^{11}$ Apatinib was approved in China as a subsequent-line therapy for patients with advanced gastric cancer. ${ }^{12}$ Promising results with a welltolerated safety profile have been reported with apatinib as first-line therapy in a multicenter, randomized, open-label, dose-finding, Phase II trial conducted in China involving treatment-naive patients with advanced HCC. ${ }^{13}$ Currently, apatinib is being evaluated as second-line therapy in a multicenter, randomized, double-blind, Phase III trial (NCT02329860) involving patients with HCC who had disease progression after sorafenib treatment or were intolerant to sorafenib. Due to paucity of prospective or retrospective comparative data for sorafenib and apatinib, we performed this study to compare the efficacy and safety of sorafenib and apatinib in the treatment of Chinese patients with intermediate and advanced HCC.

\section{Methods Study design and population}

This was a single-center, retrospective study conducted at the Guangdong General Hospital, Guangdong. Data were collected retrospectively for all consecutive patients with a diagnosis of HCC and treated with sorafenib or apatinib in the Interventional Therapy Department of Guangdong General Hospital during January 2014 to December 2016.

Patients were included in the study if they: 1) had histologically confirmed $\mathrm{HCC}$ with recidivation after radical cure or those on the oral interventional therapy of sorafenib or apatinib after systemic chemotherapy, and 2) were either BCLC stage B or C with Child-Pugh grading $<9$. Patients were excluded from the study if they had received initial treatment with sorafenib or apatinib and then changed to other targeted therapies or traditional Chinese medicine (TCM). The patients were grouped into apatinib treatment (group A) or sorafenib treatment (group S) and followed up till April 30, 2017. As apatinib was launched in the market only in October 2014, the patients in group A took the earliest drug on December 22, 2014.

The study protocol was approved by the Institutional Review Board of the Guangdong General Hospital, Guangdong, while confirming the standards of the Declaration of Helsinki and its subsequent revisions. As the use of apatinib for the treatment of $\mathrm{HCC}$ is limited to clinical trials in China, the patients were made aware of the medication and the related adverse events (AEs). The patients chose apatinib mainly because of the lower cost compared to sorafenib. The written informed consent was obtained from all the patients who chose apatinib and sorafenib.

\section{Treatment received}

The patients in the apatinib group received initial recommended dose of $750 \mathrm{mg}$ once daily (QD), which was reduced to $250 \mathrm{mg}$ QD in the case of any class 3 or $4 \mathrm{AE}$. Sorafenib was administered orally $400 \mathrm{mg}$ twice daily (BID), and dose was modified to 400 or $200 \mathrm{mg}$ QD in the case of grade 3 or 4 AEs.

\section{Study outcomes}

The patients underwent computed tomography or magnetic resonance imaging every 8-12 weeks. Efficacy was measured by progression-free survival (PFS), OS, as well as objective response rate (ORR) according to the modified response evaluation criteria in solid tumors (mRECIST) criteria. ${ }^{14}$ ORR was defined as the partial or complete response (CR) to the target drugs in the overall population. PFS was defined as the time from the first dose until the disease progression or diagnosis of new foci of disease or distant metastasis or death. 
OS was defined as the time from the first dose until death or the last follow-up.

Safety assessment of sorafenib and apatinib was reported according to the National Cancer Institute's Common Terminology Criteria for Adverse Events (CTCAE), Version 4.0. AEs that required hospitalization or prolonged hospital stay or caused crippling of self-care daily life loss of movement or those that endangered lives were classified as grade 4 , and in cases of death, were graded as grade 5 .

\section{Statistical analyses}

All statistical analyses were performed using SPSS version 22.0 (IBM Corporation, Armonk, NY, USA). Descriptive statistics were used to present the baseline, efficacy, and safety characteristics. To compare the baseline characteristics, ORR, and incidence of class 3-5 AEs between the two groups, Fisher's exact test was used. As the frequency was $>5$, chi-square test was applied to compare the PFS and the types of AEs in the two groups. Survival curves were calculated for both groups by using Kaplan-Meier (KM) methods, and the OS was calculated by using the log-rank test. A $P$-value of $<0.05$ was considered as statistically significant.

\section{Results}

\section{Baseline characteristics}

A total of $72 \mathrm{HCC}$ patients were identified based on the treatment received and categorized into apatinib group $(n=34$ patients; age range 33-78 years; 31 males) and sorafenib group ( $\mathrm{n}=38$ patients; age range $28-77$ years; 34 males). Vital details recorded as baseline characteristics including the tumor characteristics, prior infections, and treatments are presented in Table 1. No statistically significant differences were noted in any of the baseline characteristics between the two treatment groups.

Table I Baseline characteristics

\begin{tabular}{|c|c|c|c|}
\hline Characteristics & $\begin{array}{l}\text { Sorafenib group } \\
\text { (group S) }\end{array}$ & $\begin{array}{l}\text { Apatinib group } \\
\text { (group A) }\end{array}$ & $P$-value \\
\hline Total number of patients $(n)$ & 38 & 34 & \\
\hline Age (years) (mean \pm s.d.) & $28-77(50.63 \pm 10.22)$ & $33-78(53.32 \pm 12.19)$ & 0.23 \\
\hline Sex & & & 1.00 \\
\hline Male, n (\%) & $34(89.47)$ & 31 (9I.I7) & \\
\hline Female, $\mathrm{n}(\%)$ & $4(10.52)$ & $3(8.82)$ & \\
\hline Tumor number & & & 1.00 \\
\hline $\mathrm{I}-5, \mathrm{n}(\%)$ & $20(52.63)$ & $15(44.11)$ & \\
\hline$>5, n(\%)$ & $18(47.36)$ & $19(55.88)$ & \\
\hline Child-Pugh grading & & & 0.12 \\
\hline $\mathrm{A}, \mathrm{n}(\%)$ & $34(89.47)$ & $25(73.52)$ & \\
\hline $\mathrm{B}, \mathrm{n}(\%)$ & $4(10.52)$ & $9(26.47)$ & \\
\hline BCLC staging & & & 1.00 \\
\hline $\mathrm{B}, \mathrm{n}(\%)$ & $8(21.05)$ & $7(20.588)$ & \\
\hline C, n (\%) & $30(78.94)$ & $27(79.4 I I)$ & \\
\hline AFP level (ng/mL) & & & 0.27 \\
\hline Mean values \pm SD & $34,991.26 \pm 17,353.18$ & $55,975.05 \pm 12,393.88$ & \\
\hline PS scoring & & & 0.77 \\
\hline $0, \mathrm{n}(\%)$ & $8(21.05)$ & $6(17.65)$ & \\
\hline $\mathrm{I}, \mathrm{n}(\%)$ & $30(78.95)$ & $28(82.35)$ & \\
\hline Prior surgery & & & 0.25 \\
\hline Yes, n (\%) & $21(55.26)$ & $14(4 \mid .18)$ & \\
\hline No, n (\%) & $17(44.74)$ & $20(58.82)$ & \\
\hline Prior TACE therapy & & & 0.70 \\
\hline Yes, n (\%) & $35(92.10)$ & $30(88.23)$ & \\
\hline No, n (\%) & $3(7.90)$ & $4(11.76)$ & \\
\hline HBV infection & & & 1.00 \\
\hline Yes, n (\%) & 31 (8I.58) & $27(79.4 I)$ & \\
\hline No, n (\%) & 7 (18.42) & $7(20.58)$ & \\
\hline Alcohol use & & & 0.82 \\
\hline Yes, n (\%) & $18(47.37)$ & $15(44.12)$ & \\
\hline No, n (\%) & $20(52.63)$ & $19(55.88)$ & \\
\hline Smoking & & & 1.00 \\
\hline Yes, n (\%) & $15(39.47)$ & $13(38.24)$ & \\
\hline No, n (\%) & $23(60.53)$ & $21(61.76)$ & \\
\hline
\end{tabular}

Abbreviations: AFP, alpha-fetoprotein; BCLC, Barcelona-Clinic Liver Cancer; HBV, hepatitis B virus; PS, performance status; s.d., standard deviation; TACE, transcatheter arterial chemoembolization. 
Table 2 Response rates by mRECIST criteria

\begin{tabular}{llll}
\hline Characteristics & $\begin{array}{l}\text { Sorafenib } \\
\text { group } \\
\text { (group S), } \\
\mathbf{n}=\mathbf{3 8}\end{array}$ & $\begin{array}{l}\text { Apatinib } \\
\text { group } \\
\text { (group A), } \\
\mathbf{n}=\mathbf{3 4}\end{array}$ & P-value \\
\hline CR, n & $\mathrm{I}$ & 0 & \\
PR, n & 5 & 6 & \\
SD, $\mathrm{n}$ & 10 & 7 & 0.829 \\
ORR (CR + PR/N) (\%) & 15.78 & 17.64 & 0.750 \\
DCR (CR + PR + SD/N) (\%) & 42.10 & 38.23 & 0.031 \\
mPFS (months), median & 7.39 & 4.79 & \\
\hline
\end{tabular}

Abbreviations: $\mathrm{CR}$, complete response; $\mathrm{DCR}$, disease control rate; mPFS, median progression-free survival; N, number of patients in the group; ORR, overall response rate; $P R$, partial response; $m R E C I S T$, modified response evaluation criteria in solid tumors; SD, stable disease.

\section{Response rates and survival}

Among the 38 patients treated with sorafenib, 1 patient had $\mathrm{CR}, 5$ patients had partial response (PR), and 10 patients had stable disease (SD). The ORR was $15.78 \%$, and the disease control rate (DCR) was $42.1 \%$. Sorafenib-treated patients had median PFS duration of 7.39 months. Of the 34 apatinib-treated patients, 6 patients had PR and 7 patients had SD with no cases of CR. The ORR was $17.64 \%$, the DCR was $38.2 \%$, and the mPFS was 4.79 months (Table 2). There were no statistically significant differences in ORR ( $P=0.833)$ and DCR $(P=0.75)$ as assessed by the Fisher's exact test in the sorafenib and apatinib groups. The mPFS was significantly longer in the sorafenib group than in the apatinib group $(P=0.031)$ (Table 2$)$.

The median OS of the complete study population was 9.04 months (95\% confidence interval [CI]: 7.7-10.37). Median OS in patients treated with sorafenib was significantly greater compared with that in patients treated with apatinib (10.4 months [95\% CI: 8.3-12.47] vs 7.18 months [95\% CI: 5.97-8.4], respectively, $P=0.011$ ) (Figure 1).

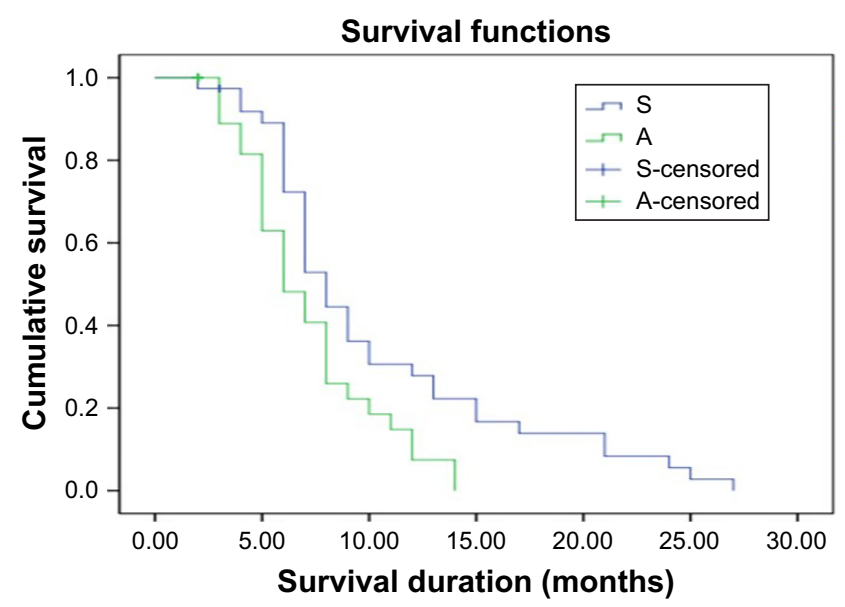

Figure I KM curve for OS after sorafenib and apatinib treatments. Abbreviations: A, apatinib; KM, Kaplan-Meier; OS, overall survival; S, sorafenib.

\section{Safety analysis}

Table 3 presents the overall safety profile of both the treatment groups as per the grades. Compared with apatinib, sorafenib group had greater overall incidence of hand and foot syndrome reaction (HFSR, 13 vs 10; diarrhea, 13 vs 2; anorexia, 8 vs 2; alopecia, 8 vs 0 , respectively). On the contrary, apatinib group reported greater incidence of hypertension (17 vs 7), elevated transaminase levels (14 vs 4), and proteinuria (15 vs 3). Serious AEs (grade 3-5) were reported for HFSR $(n=2,15.3 \%)$, hypertension $(n=1$, $14.28 \%)$, diarrhea $(n=1,7.69 \%)$, elevated transaminase $(n=1$, $25 \%)$, and anorexia $(n=1,12.5 \%)$ in the sorafenib group. For apatinib, grade 3-5 AEs were reported for HFSR ( $n=1,10 \%)$, elevated transaminase $(n=2,14.28 \%)$, and proteinuria $(n=2$, $13.33 \%$ ) indicating less incidence of serious AEs (SAEs) with apatinib. A significant difference was observed between the AEs in the sorafenib and apatinib groups $(P<0.001)$. HFSR, hypertension, diarrhea, and loss of appetite were reported within the first 4 weeks of starting sorafenib, whereas in the apatinib group, HFSR and proteinuria were reported in the first 4 weeks of taking the first dose. All the events were controlled by reducing the dose of the study drugs.

\section{Discussion}

Due to the aggressive nature and fast progression of HCC, $>20 \%$ of the cases are diagnosed only in advancedstage HCC. ${ }^{15,16}$ Currently, sorafenib, a VEGFR and PDGFR inhibitor, is the only approved, and the gold standard, treatment for advanced HCC, which predominantly acts by inhibiting tumor cell proliferation, thereby delaying disease progression. ${ }^{3,8,17}$ To the best of our knowledge, no previous study has reported the comparison between sorafenib and apatinib (a new multikinase inhibitor) and ours is the first study comparing the efficacy and safety of the two drugs in Chinese patients with intermediate and advanced HCC.

Published evidence presents the greater efficacy of sorafenib compared with placebo in multiple randomized controlled trials (RCTs). A global, placebo-controlled, Phase III trial spanning across 121 centers in 21 countries of Europe, North America, South America, and Australia had reported a significant OS difference between sorafenib and placebo (10.7 vs 7.9 months; hazard ratio [HR] 0.69; 95\% CI: $0.55-0.87 ; P<0.001)$. Also, a significantly higher median time to radiologic progression was reported in the sorafenib group in comparison to placebo (5.5 vs 2.8 months; HR 0.58 ; 95\% CI: $0.45-0.74 ; P<0.001) .{ }^{8}$ Another large-scale study performed in 23 centers in the Asia-Pacific countries reported a median OS of 6.5 months and a time to progression (TTP) of 2.8 months in the sorafenib group in comparison to an OS 
Table 3 Post-treatment AEs

\begin{tabular}{|c|c|c|c|c|c|c|}
\hline \multirow[t]{2}{*}{ AE } & \multicolumn{3}{|c|}{ Sorafenib $(n=38)$} & \multicolumn{3}{|c|}{ Apatinib $(n=34)$} \\
\hline & $\begin{array}{l}\text { All AEs, } \\
\text { n (\%) }\end{array}$ & $\begin{array}{l}\text { Grade I-2, } \\
\text { n (\%) }\end{array}$ & $\begin{array}{l}\text { Grade 3-5, } \\
\text { n (\%) }\end{array}$ & $\begin{array}{l}\text { All AEs, } \\
\text { n (\%) }\end{array}$ & $\begin{array}{l}\text { Grade I-2, } \\
\text { n (\%) }\end{array}$ & $\begin{array}{l}\text { Grade 3-5, } \\
\text { n (\%) }\end{array}$ \\
\hline HFS & $13(34.21)$ & II (29.0) & $2(5.3)$ & $10(29.4 I)$ & $9(26.47)$ & I (2.94) \\
\hline Hypertension & $7(18.42)$ & $6(15.78)$ & I (2.63) & $17(50)$ & $17(50)$ & 0 \\
\hline Diarrhea & $13(34.21)$ & $12(31.57)$ & I (2.63) & $2(5.88)$ & $2(5.88)$ & 0 \\
\hline Elevated transaminase & $4(10.52)$ & $3(7.9)$ & I (2.63) & $14(4 \mid .17)$ & $12(35.29)$ & $2(5.88)$ \\
\hline Anorexia & $8(21.05)$ & $7(18.42)$ & I (2.63) & $2(5.88)$ & $2(5.88)$ & 0 \\
\hline Proteinuria & $3(7.89)$ & 3 (7.89) & 0 & $15(44.11)$ & $13(38.23)$ & $2(5.88)$ \\
\hline Alopecia & $8(21.05)$ & $8(21.05)$ & 0 & 0 & 0 & 0 \\
\hline
\end{tabular}

Abbreviations: AEs, adverse events; HFS, hand and foot syndrome.

of 4.2 months and a TTP of 1.4 months in the placebo group. ${ }^{3}$ In addition, none of the other antiangiogenic tyrosine kinase inhibitors including sunitinib, ${ }^{18}$ linifanib,${ }^{4}$ brivanib, ${ }^{19}$ and dovitinib $^{20}$ were found superior to sorafenib in Phase II and III trials as first-line therapies in patients with advanced HCC. Thus, these trials confirmed the efficacy aspect of sorafenib in advanced $\mathrm{HCC}$ in a wider range of population. Despite the efficacy of sorafenib reported in multiple studies, $\sim 60 \%-70 \%$ of advanced HCC patients eventually develop disease progression after sorafenib treatment. ${ }^{10}$ Sorafenib therapy has also been frequently challenged by the onset of SAEs and liver failure, thereby leading to treatment noncompliance along with losing significant therapeutic benefits. ${ }^{21-23}$

Apatinib (YN968D1; Advenchen Laboratories, LLC, Northridge, CA, USA) is among the latest antiangiogenic agent with 10 times the VEGFR-binding activity of sorafenib ${ }^{24,25}$ with encouraging preclinical and clinical results for many solid tumors. It acts via the VEGF pathway, inhibiting VEGFR-2 for targeting the intracellular adenosine triphosphata-binding site of the receptor and inhibiting VEGF-stimulated endothelial cell migration and proliferation, reduces the tumor microvascular density, and promotes apoptosis. ${ }^{11,26,27}$ Use of apatinib has been explored as first-line therapy for advanced HCC in a Phase II RCT ${ }^{10}$ with longer PFS and OS reported for $850 \mathrm{mg}$ apatinib than $750 \mathrm{mg}$ apatinib (4.2 and 3.3 months) but with similar OS (9.7 and 9.8 months, respectively). Apatinib's efficacy has also been explored in combination with TACE. A comparative study assessing the efficacy of TACE combined with apatinib and TACE alone in the treatment of intermediate and advanced HCC reported a higher median PFS of 12.5 months in apatinib with TACE group in comparison to 6 months in TACE alone group. ${ }^{28}$

In our study, a significantly longer mPFS (7.39 vs 4.79 months, $P=0.031)$ and median OS (10.4 vs 7.18 months, $P=0.011$ ) was observed in the sorafenib group in comparison to the apatinib group. The findings of our study were consistent with the previous findings of significant improvement in OS and PFS $;^{3,4,8,20}$ furthermore, median PFS and OS durations in our study were comparatively higher than placebocontrolled RCTs of sorafenib; however, limited information is available on apatinib in HCC patients. A multicenter, randomized, double-blind, Phase III trial (NCT02329860) is ongoing that intends to evaluate the efficacy and safety of apatinib in patients with advanced liver cancer who have progressed on systemic therapy.

In a recent case report, a 45-year-old male patient with BCLC stage $3 \mathrm{HCC}$ was treated with $500 \mathrm{mg}$ apatinib combined with transhepatic arterial chemotherapy and embolization and chemotherapy. The patient had PR with a notable reduction in the level of serum alpha-fetoprotein (AFP) from 60,500 to $12.7 \mathrm{ng} / \mathrm{mL}$ and PFS of $>8$ months, thereby indicating apatinib as a superior choice for HCC patients. ${ }^{29}$ The median PFS and OS of apatinib reported in the current study (4 and 10.4 months) were similar to those reported in heavily pretreated patients with nontriple-negative metastatic breast cancer (4 and 10.3 months). ${ }^{30}$ Similarly, a Phase II study in metastatic gastric cancer patients had reported an improved PFS of 3.67 months $^{31}$ and a Phase III study in chemotherapy-refractory advanced or metastatic adenocarcinoma of the stomach or gastroesophageal junction had reported an improved PFS of 2.6 months and an OS of 6.5 months in apatinib-treated patients. ${ }^{32}$ The findings from our study and previous studies confirm the efficacy of apatinib in multiple tumor types and as they showed similar efficacy in terms of survival improvement. In our study, it was evident that sorafenib achieved a better ORR ( 0.17 vs $0.15 \%)$ and DCR $(0.42$ vs $0.38 \%)$ in comparison to the apatinib group. The ORR reported in our study was greater than that reported in a Phase III trial, where the ORR was just $6.1 \%$ for sorafenib in comparison to $10.1 \%$ exhibited by linifanib. ${ }^{4}$ Compared to our study, the SHARP sub-analysis by Bruix et $\mathrm{al}^{33}$ reported a higher DCR with sorafenib in HCV-infected (44.2\%), Hepatitis B virus-positive (34.4\%), and alcohol-related HCC patients $(54.4 \%)$. The DCR and ORR of apatinib reported in 
the current study were lower than those reported in heavily pretreated patients with nontriple-negative metastatic breast cancer (66.7 and $16.7 \%){ }^{30}$

There was no difference in the incidence rate of SAEs, with no related death reported in either of the two groups. In our study, the common AEs reported in the apatinib group included proteinuria (44\%), hypertension (50\%), elevated transaminase (41\%), and hand and foot syndrome (HFS) (29.4\%). AEs reported were similar to those reported previously; however, the morbidity was lower than that reported in the Phase III clinical test of gastrointestinal tumors ${ }^{32}$ and this may be due to the different cancer types or small sample sizes or because of poor liver functions. The AEs reported in the sorafenib group included HFSR (34\%), diarrhea (34\%), anorexia (21\%), and alopecia (21\%), and the results were similar to that observed in the SHARP study. ${ }^{3}$ Overall, apatinib showed better safety profile and lower number of grade 3-5 AEs, which could probably be associated with selective inhibition of VEGF. ${ }^{34}$

In a clinical practice, there is variation in the recommended dosing of sorafenib and it is mostly prescribed based on the baseline Child-Pugh status, performance status, comorbidities, and the treating physician's preference. The study by Al-Rajabi et a ${ }^{35}$ has reported safe and efficient outcomes with full dosing $(800 \mathrm{mg} /$ day $)$ of sorafenib in comparison to the reduced dose in HCC patients with liver dysfunction.

Currently, limited data are available on the effect of dosing on the efficacy of apatinib, especially in HCC patients. Though the tested and recommended dose of apatinib is $750-850 \mathrm{mg} /$ day, there are retrospective studies and case reports that have demonstrated significant efficacy and safety at a lower dose of $250-500 \mathrm{mg} /$ day. ${ }^{29,36}$ However, further well-controlled studies are required to validate the benefit of altered dosing in HCC patients. Our study strengths included 1) no loss of patients at follow-up, and 2) being the first reported study from China comparing the effectiveness of these two antiangiogenic drugs in patients with intermediate or advanced HCC. Other than the strengths, our study had a few limitations so findings must be interpreted cautiously. First, our study had a retrospective design, which might have led to unintentional bias during data extraction and reporting. Second, the patients were selected from a single center and in small number; therefore, the results might be difficult to generalize in a larger number of patients. Third, the decision to treat with sorafenib or apatinib was made by the treating physicians who might be prone to selection bias due to their belief in the superiority of one of the treatments.

\section{Conclusion}

Though generally well-tolerated, apatinib did not have improved activity over sorafenib in patients with intermediate or advanced HCC. As the ORR and DCR were similar, apatinib can be of potential benefit, especially for those who have progressed on sorafenib therapy. Further prospective, randomized controlled multicenter studies are needed to verify the findings.

\section{Acknowledgment}

We would like to thank Dr Dhanya Mukundan and Mr Karan Sharma from Indegene, Bangalore, India, for providing medical writing support and technical assistance in the preparation of this manuscript, as funded by Bayer Pharmaceutical Company.

\section{Author contributions}

All authors contributed toward data analysis, drafting and critically revising the paper, gave final approval of the version to be published, and agree to be accountable for all aspects of the work.

\section{Disclosure}

The authors report no conflicts of interest in this work.

\section{References}

1. Feng $\mathrm{X}, \mathrm{Xu} \mathrm{R}, \mathrm{Du} \mathrm{X}$, et al. Combination therapy with sorafenib and radiofrequency ablation for BCLC stage 0-B1 hepatocellular carcinoma: a multicenter retrospective cohort study. Am J Gastroenterol. 2014; 109(12):1891-1899.

2. Muhammad A, Dhamija M, Vidyarthi G, et al. Comparative effectiveness of traditional chemoembolization with or without sorafenib for hepatocellular carcinoma. World J Hepatol. 2013;5(7):364-371.

3. Cheng A-L, Kang Y-K, Chen Z, et al. Efficacy and safety of sorafenib in patients in the Asia-Pacific region with advanced hepatocellular carcinoma: a phase III randomised, double-blind, placebo-controlled trial. Lancet Oncol. 2009;10(1):25-34.

4. Cainap C, Qin S, Huang W-T, et al. Linifanib versus sorafenib in patients with advanced hepatocellular carcinoma: results of a randomized phase III trial. J Clin Oncol. 2015;33(2):172-179.

5. Wu Q, Qin SK. Features and treatment options of Chinese hepatocellular carcinoma. Chin Clin Oncol. 2013;2(4):38.

6. Wilhelm S, Carter C, Lynch M, et al. Discovery and development of sorafenib: a multikinase inhibitor for treating cancer. Nat Rev Drug Discov. 2006;5(10):835-844.

7. Yu SJ. A concise review of updated guidelines regarding the management of hepatocellular carcinoma around the world: 2010-2016. Clin Mol Hepatol. 2016;22(1):7-17.

8. Llovet JM, Ricci S, Mazzaferro V, et al; SHARP Investigators Study Group. Sorafenib in advanced hepatocellular carcinoma. N Engl J Med. 2008;359(4):378-390.

9. Park J-W, Chen M, Colombo M, et al. Global patterns of hepatocellular carcinoma management from diagnosis to death: the BRIDGE Study. Liver Int. 2015;35(9):2155-2166.

10. Gomaa A, Waked I. Management of advanced hepatocellular carcinoma: review of current and potential therapies. Hepatoma Res. 2017; $3: 112-122$. 
11. Tian S, Quan H, Xie C, et al. YN968D1 is a novel and selective inhibitor of vascular endothelial growth factor receptor-2 tyrosine kinase with potent activity in vitro and in vivo. Cancer Sci. 2011;102(7): 1374-1380.

12. Zhang H. Apatinib for molecular targeted therapy in tumor. Drug Des Devel Ther. 2015;9:6075-6081.

13. Qin S. Apatinib in Chinese patients with advanced hepatocellular carcinoma: a phase II randomized, open-label trial. J Clin Oncol. 2014; 32(supp1 5):abstract4019.

14. Lencioni R, Llovet JM. Modified RECIST (mRECIST) assessment for hepatocellular carcinoma. Semin Liver Dis. 2010;30(1):52-60.

15. Pinter M, Hucke F, Graziadei I, et al. Advanced-stage hepatocellular carcinoma: transarterial chemoembolization versus sorafenib. Radiology. 2012;263(2):590-599.

16. Hucke F, Sieghart W, Schöniger-Hekele M, Peck-Radosavljevic M, Müller C. Clinical characteristics of patients with hepatocellular carcinoma in Austria - is there a need for a structured screening program? Wien Klin Wochenschr. 2011;123(17-18):542-551.

17. Wilhelm SM, Carter C, Tang L, et al. BAY 43-9006 exhibits broad spectrum oral antitumor activity and targets the RAF/MEK/ERK pathway and receptor tyrosine kinases involved in tumor progression and angiogenesis. Cancer Res. 2004;64(19):7099-7109.

18. Cheng A-L, Kang Y-K, Lin D-Y, et al. Sunitinib versus sorafenib in advanced hepatocellular cancer: results of a randomized phase III trial. J Clin Oncol. 2013;31(32):4067-4075.

19. Johnson PJ, Qin S, Park J-W, et al. Brivanib versus sorafenib as firstline therapy in patients with unresectable, advanced hepatocellular carcinoma: results from the randomized phase III BRISK-FL study. J Clin Oncol. 2013;31(28):3517-3524.

20. Cheng A-L, Thongprasert S, Lim HY, et al. Randomized, open-label phase 2 study comparing frontline dovitinib versus sorafenib in patients with advanced hepatocellular carcinoma. Hepatology. 2016;64(3): 774-784.

21. Reig M, Rimola J, Torres F, et al. Postprogression survival of patients with advanced hepatocellular carcinoma: rationale for second-line trial design. Hepatology. 2013;58(6):2023-2031.

22. Personeni N, Bozzarelli S, Pressiani T, et al. Usefulness of alphafetoprotein response in patients treated with sorafenib for advanced hepatocellular carcinoma. J Hepatol. 2012;57(1):101-107.

23. Iavarone M, Cabibbo G, Piscaglia F, et al; SOFIA (SOraFenib Italian Assessment) Study Group. Field-practice study of sorafenib therapy for hepatocellular carcinoma: a prospective multicenter study in Italy. Hepatology. 2011;54(6):2055-2063.
24. Peng S, Li J, Zhang Y, Xu L, Kuang M, Chen M. Apatinib inhibited cell proliferation directly through suppressing VEGF signaling in extrahepatic bile duct carcinoma. Gastroenterology. 2015;148(4): S1026-S1027.

25. Li J, Zhao X, Chen L, et al. Safety and pharmacokinetics of novel selective vascular endothelial growth factor receptor-2 inhibitor YN968D1 in patients with advanced malignancies. BMC Cancer. 2010;10:529.

26. Peng H, Zhang Q, Li J, et al. Apatinib inhibits VEGF signaling and promotes apoptosis in intrahepatic cholangiocarcinoma. Oncotarget. 2016;7(13):17220-17229.

27. Peng S, Zhang Y, Peng $H$, et al. Intracellular autocrine VEGF signaling promotes EBDC cell proliferation, which can be inhibited by apatinib. Cancer Lett. 2016;373(2):193-202.

28. Lu W, Jin X-L, Yang C, et al. Comparison of efficacy between TACE combined with apatinib and TACE alone in the treatment of intermediate and advanced hepatocellular carcinoma: a single-center randomized controlled trial. Cancer Biol Ther. 2017;18(6):433-438.

29. Kou P, Zhang Y, Shao W, et al. Significant efficacy and well safety of apatinib in an advanced liver cancer patient: a case report and literature review. Oncotarget. 2017;8(12):20510-20515.

30. $\mathrm{Hu}$ X, Cao J, Hu W, et al. Multicenter phase II study of apatinib in nontriple-negative metastatic breast cancer. BMC Cancer. 2014;14:820.

31. Li J, Qin S, Xu J, et al. Apatinib for chemotherapy-refractory advanced metastatic gastric cancer: results from a randomized, placebo-controlled, parallel-arm, phase II trial. J Clin Oncol. 2013;31(26):3219-3225.

32. Li J, Qin S, Xu J, et al. Randomized, double-blind, placebo-controlled phase III trial of apatinib in patients with chemotherapy-refractory advanced or metastatic adenocarcinoma of the stomach or gastroesophageal junction. J Clin Oncol. 2016;34(13):1448-1454.

33. Bruix J, Raoul J-L, Sherman M, et al. Efficacy and safety of sorafenib in patients with advanced hepatocellular carcinoma: subanalyses of a phase III trial. J Hepatol. 2012;57(4):821-829.

34. Roviello G, Ravelli A, Polom K, et al. Apatinib: a novel receptor tyrosine kinase inhibitor for the treatment of gastric cancer. Cancer Lett. 2016;372(2):187-191.

35. Al-Rajabi R, Patel S, Ketchum NS, et al. Comparative dosing and efficacy of sorafenib in hepatocellular cancer patients with varying liver dysfunction. J Gastrointest Oncol. 2015;6(3):259-267.

36. Kong Y, Sun L, Hou Z, et al. Apatinib is effective for treatment of advanced hepatocellular carcinoma. Oncotarget. 2017;8(62):105596-105605.
OncoTargets and Therapy

\section{Publish your work in this journal}

OncoTargets and Therapy is an international, peer-reviewed, open access journal focusing on the pathological basis of all cancers, potential targets for therapy and treatment protocols employed to improve the management of cancer patients. The journal also focuses on the impact of management programs and new therapeutic agents and protocols on

\section{Dovepress}

patient perspectives such as quality of life, adherence and satisfaction. The manuscript management system is completely online and includes a very quick and fair peer-review system, which is all easy to use. Visit http://www.dovepress.com/testimonials.php to read real quotes from published authors. 